10. НУРГЕС

\title{
МОДЕЛЬ СОСТОЯНИЯ ДИСКРЕТНОЙ СИСТЕМЫ В ОРТОГОНАЛЬНОМ БАЗИСЕ
}

\author{
(Представил Н. Алумяэ)
}

\section{1. Введение}

Во многих задачах фильтрации $\left[{ }^{1,2}\right]$ и идентификации $\left[{ }^{3}\right]$ используют разложение по ортогональным многочленам. При этом динамнческая система определяется своей импульсной характеристикой $\left[{ }^{1,3}\right]$ или же передаточной функцией $\left[{ }^{2}\right]$. Так как основой современной теории управления, а также задач идентификации и фильтрации, является модель состояния, то представляет интерес вывести модель состояния в базисе ортогональных многочленов. Первые работы такого рода посвящены многочленам Лагерра $\left[{ }^{4,5}\right]$.

В данной работе изучаются экспоненциально-степенные многочлены, предложенные В. П. Перовым для решения задачи оптимальной фильтрации [ $\left.{ }^{1}\right]$. Выведена рекуррентная формула между этими многочленами, позволившая построить модель состояния в экспоненциальностепенном базисе. Это новое представление системы включает свободно выбираемый параметр, изменением которого можно в широких пределах варьировать свойства системы. При этом модель состояния остается управляемой и наблюдаемой, если только исходная система управляема и наблюдаема. При переходе в экспоненциально-степенной базис порядок системы и нулевое начальное состояние не изменяются.

\section{2. Экспоненциально-степенные многочлены}

Экспоненциально-степенные многочлены дискретного переменного определяются следующим образом [ $\left.{ }^{1}\right]$ :

$$
l_{k}(t)=k ! \sum_{j=0}^{k}\left(1-\mathrm{e}^{a}\right)^{j}\left(\begin{array}{l}
k \\
j
\end{array}\right)\left(\begin{array}{l}
t \\
j
\end{array}\right),
$$

где $\left(\begin{array}{l}k \\ j\end{array}\right)$ - биномиальный коэффициент, $a \in[0, \infty)$.

Многочлены $l_{k}(t)$ ортогональны относительно экспоненциального веса

$$
\sum_{t=0}^{\infty} \omega(t) l_{k}(t) l_{m}(t)=\left(\gamma_{k}\right)^{-1} \delta_{k m},
$$

где $\omega(t):=\mathrm{e}^{-a t}, \gamma_{k}=(k !)^{-2} \mathrm{e}^{-(k+1) a}\left(\mathrm{e}^{a}-1\right), \delta_{k m}-$ символ Кронекера.

Многочлены $l_{k}(t)$ можно определить также с помощью оператора разности

$$
l_{k}(t)=k ! \mathrm{e}^{(k+t) a} \Delta^{k}\left[\mathrm{e}^{-a t}\left(\begin{array}{c}
t \\
k
\end{array}\right)\right] .
$$


$\overline{\mathrm{B}}$ частностии,

$$
\begin{aligned}
& l_{0}(t)=1, \\
& l_{1}(t)=1-c t, \quad c=\mathrm{e}^{a}-1, \\
& l_{2}(t)=2-4 c t+c^{2} t(t-1), \\
& l_{3}(t)=6-18 c t+9 c^{2} t(t-1)-c^{3} t(t-1)(t-2) .
\end{aligned}
$$

Они удовлетворяют рекуррентной формуле

$$
l_{k+1}(t)=\left[(2 k+1)+(k-t)\left(\mathrm{e}^{a}-1\right)\right] l_{k}(t)-k^{2} \mathrm{e}^{a} l_{k-1}(t), \quad\left(l_{-1}(t)=0\right) .
$$

Для вывода модели состояния дискретной системы в экспоненциальностепенном базисе понадобится формула, определяющая многочлены $l_{k}(t+1), k=0,1,2, \ldots$, линейной комбинацией многочленов $l_{i}(t)$, $i=0, \ldots, k$,

$$
l_{k}(t+1)=\sum_{i=0}^{k} q_{i k} l_{k}(t)
$$

По определению (1)

$$
l_{k}(t+1)=k ! \sum_{j=0}^{k}(-c)^{j}\left(\begin{array}{c}
k \\
j
\end{array}\right)\left(\begin{array}{c}
t+1 \\
j
\end{array}\right) .
$$

Учитывая свойство биномиальных коэффициентов

$$
\left(\begin{array}{c}
t+1 \\
j
\end{array}\right)=\left(\begin{array}{l}
t \\
j
\end{array}\right)+\left(\begin{array}{c}
t \\
j-1
\end{array}\right)
$$

получим

$$
l_{k}(t+1)=l_{k}(t)+k ! \sum_{j=1}^{k}(-c)^{j}\left(\begin{array}{l}
k \\
j
\end{array}\right)\left(\begin{array}{c}
t \\
j-1
\end{array}\right)
$$

T. e.

$$
q_{k k}=1 \text {. }
$$

Покажем, что найдутся коэффициенты $q_{i k}, i=0, \ldots, k-1$, такие, что

$$
\sum_{i=0}^{k-1} q_{i k} l_{k}(t)=k ! \sum_{j=0}^{k-1}(-c)^{j+1}\left(\begin{array}{c}
k \\
j-1
\end{array}\right)\left(\begin{array}{l}
t \\
j
\end{array}\right) \text {. }
$$

Использовав определение (1) и изменив порядок суммирования, получим

$$
\begin{aligned}
\sum_{i=0}^{k-1} q_{i k} l_{k}(t) & =\sum_{i=0}^{k-1} q_{i k} i ! \sum_{j=0}^{i}(-c)^{j}\left(\begin{array}{c}
i \\
j
\end{array}\right)\left(\begin{array}{l}
t \\
j
\end{array}\right)= \\
& =\sum_{j=0}^{k-1}(-c)^{j}\left(\begin{array}{l}
t \\
j
\end{array}\right) \sum_{i=j}^{k-1} q_{i k} i !\left(\begin{array}{l}
i \\
j
\end{array}\right) .
\end{aligned}
$$

Выбирая теперь коэффициенты $q_{i k}, i=0, \ldots, k-1$, следующим образом

$$
q_{i k}=-c k ! / i
$$

и учитывая еще свойство биномиальных коэффициентов

$$
\sum_{i=j}^{k-1}\left(\begin{array}{l}
i \\
j
\end{array}\right)=\left(\begin{array}{c}
k \\
j+1
\end{array}\right) \text {, }
$$


получим равенство (5). Значит, формула (3) выполняется, если выбирать коэффициенты $q_{i k}, i=0, \ldots, k$, по (4) и (6), т. е.

$$
l_{k}(t+1)=l_{k}(t)-c \sum_{i=0}^{k-1} \frac{k !}{i !} l_{i}(t) \text {. }
$$

\section{3. Модель состояния в экспоненциально-степенном базисе}

Рассмотрим дискретную многомерную динамическую систему

$$
\begin{aligned}
x(t+1) & =F x(t)+G u(t), \\
y(t) & =H x(t), \quad t=0,1,2, \ldots,
\end{aligned}
$$

где $x-n$-вектор состояния, $u-m$-вектор управляющих воздействий, $y$ - $p$-вектор выходных переменных системы. Матрицы $F, G, H$ имеют размерность $n \times n, n \times m$ и $p \times n$ соответственно.

Экспоненциально-степенные многочлены $l_{k}(t)$ составляют полную ортогональную систему, и любая последовательность вещественных чисел $f(t), t=0,1,2, \ldots$, может быть представлена бесконечной суммой

$$
f(t)=\sum_{k=0}^{\infty} f_{k} l_{k}(t)
$$

причем

$$
f_{k}=\sum_{t=0}^{\infty} \omega(t) \gamma_{k} f(t) l_{k}(t)
$$

Умножим теперь обе части уравнений (8) и (9) на $\omega(t) \gamma_{k} l_{k}(t)$ и суммируем их по $t=0,1,2, \ldots$. Получим соотношения между векторами коэффициентов разложения дискретных функций $x(t), u(t)$ и $y(t)$ по экспоненциально-степенным многочленам $l_{k}(t)$ :

$$
\begin{aligned}
& \bar{x}_{k}=F x_{k}+G u_{k}, \\
& y_{k}=H x_{k},
\end{aligned}
$$

где

$$
\bar{x}_{k}=\sum_{t=0}^{\infty} \omega(t) \gamma_{k} l_{k}(t) x(t+1) .
$$

На основании формул (10) и (7) получим следующее выражение для коэффициентов $\bar{x}_{k}$ :

$$
\bar{x}_{k}=\sum_{t=0}^{\infty} \omega(t) \gamma_{k} l_{k}(t) \sum_{j=0}^{\infty} x_{j} l_{j}(t)-\left(\mathrm{e}^{\alpha}-1\right) \sum_{t=0}^{\infty} \omega(t) \gamma_{k} l_{k}(t) \sum_{j=0}^{\infty} x_{j} \sum_{i=0}^{j-1} \frac{k !}{i !} l_{i}(t),
$$

а учитывая еще свойство ортогональности многочленов $l_{k}(t)(2)$, можем записать

$$
\bar{x}_{k}=x_{k}-\left(\mathrm{e}^{a}-1\right) \sum_{j=1}^{\infty} x_{k+j}
$$

Из уравнения (14) выведем соотношение между коэффициентами $\bar{x}_{k}$ и $x_{k}$

$$
\bar{x}_{k}-\bar{x}_{k+1}=x_{k}-\mathrm{e}^{a} x_{k+1} .
$$

Вернемся к уравнениям (12)- и (13). На основании формул (15) и (12) имеем 


$$
\left(F-\mathrm{e}^{a} I\right) x_{k+1}+G u_{k+1}=(F-I) x_{k}+G u_{k} .
$$

Обозначив

$$
z_{k}=\left(F-\mathrm{e}^{a} I\right) x_{k}+G u_{k},
$$

получим из (16) и (13) уравнения состояния между коэффициентами разложения $u_{k}$ и $y_{k}, k^{\prime}=0,1,2, \ldots$,

$$
\begin{aligned}
z_{k+\mathrm{t}} & =A z_{k}+B u_{k}, \\
y_{k} & =C z_{k}+D u_{k},
\end{aligned}
$$

где

$$
\begin{aligned}
& A=(F-I)\left(F-\mathrm{e}^{a} I\right)^{-1}, \\
& B=\left(1-\mathrm{e}^{a}\right)\left(F-\mathrm{e}^{a} I\right)^{-1} G, \\
& C=H\left(F-\mathrm{e}^{a} I\right)^{-1}, \\
& D=-H\left(F-\mathrm{e}^{\alpha} I\right)^{-1} G .
\end{aligned}
$$

Очевидно, что для устойчивой $F$ обратная матрица $\left(F-\mathrm{e}^{a} I\right)^{-1}$ существует, так как $a \in[0, \infty)$. Назовем уравнения (17) и (18) моделью состояния системьг в экспоненциально-степенном базисе.

Найдем начальное состояние $z_{0}$ модели $(17)-(18)$. По определению

$$
z_{0}=\left(F-\mathrm{e}^{a} I\right) x_{0}+G u_{0}
$$

или с учетом преобразования (11) и уравнения (8)

$$
z_{0}=\gamma_{0} \sum_{t=0}^{\infty} \omega(t)\left[x(t-1-1)-\mathrm{e}^{a} x(t)\right] l_{0}(t) .
$$

Нетрудно видеть, что при суммировании выпадают все слагаемые, кроме одного, а именно

$$
z_{0}=\left(1-\mathrm{e}^{a}\right) x(0) .
$$

Значит, $z_{0}=0$, если $x(0)=0$.

\section{4. Качественный анализ}

По фंормуле (19) характеристические числа матриц $A$ и $F$ связаны соотношением

$$
\lambda_{i}(A)=\left(\lambda_{i}(F)-1\right) /\left(\lambda_{i}(F)-\mathrm{e}^{a}\right), \quad i=1, \ldots, n,
$$

откуда непосредственно следует, что модель состояния в экспоненциально-степенном базисе устойчива, если только исходная система устойчива, т. е. $\left|\lambda_{i}(A)\right|<1$, если $\left|\lambda_{i}(F)\right|<1$, так как $\mathrm{e}^{a}>1$, $a \in[0, \infty)$.

Интересно отметить, что

$$
\begin{array}{ll}
\lambda_{i}(A) \rightarrow 0, & \text { если } \lambda_{i}(F) \rightarrow 1, \\
\lambda_{i}(A)=\mathrm{e}^{-a}, & \text { если } \lambda_{i}(F)=0, \\
\lambda_{i}(A) \rightarrow 2 /\left(1+-\mathrm{e}^{a}\right), & \text { если } \lambda_{i}(F) \rightarrow-1 .
\end{array}
$$

Вещественные характеристические числа исходной системы остаются вещественными и в экспоненциально-степенном базисе. При этом 


$$
\lambda_{i}(A)=\lambda_{i}(F)=\lambda_{i} \text {, если } \mathrm{e}^{a}=\mathrm{e}^{a_{o}}=\lambda_{i}^{-1}\left(\lambda_{i}^{2}-\lambda_{i}+1\right) .
$$

Если $a>a_{0}$, то $\lambda_{i}(A)<\lambda_{i}(F)$, а если $a<a_{0}$, то $\lambda_{i}(A)>\lambda_{i}(F)$.

Можно показать, что $\lambda_{i}(A)$ имеет неотрицательную вещественную составляющую и $\left|\lambda_{i}(A)\right| \leqslant 2 /\left(1+\mathrm{e}^{a}\right)$.

Пусть $\lambda_{i}(F)=r(\cos \varphi-i-j \sin \varphi)$. Тогда

$$
\lambda_{i}(A)=(r \cos \varphi-1-i-j \sin \varphi) /\left(r \cos \varphi-\mathrm{e}^{a}+j \sin \varphi\right)
$$

и

$$
\left|\lambda_{i}(A)\right|^{2}=\left(r^{2}-2 r \cos \varphi-1-1\right) /\left(r^{2}-2 \mathrm{e}^{a} r \cos \varphi+\mathrm{e}^{2 a}\right) .
$$

Покажем, что для устойчивой исходной системы $(r \leqslant 1)$

$$
\left|\lambda_{i}(A)\right|^{2} \leqslant 4 /\left(1+\mathrm{e}^{a}\right)^{2},
$$

T. e.

$$
4\left(r^{2}+\mathrm{e}^{2 a}\right)-\left(r^{2}+1\right)\left(1+\mathrm{e}^{a}\right)^{2}+2 r \cos \varphi\left(1-\mathrm{e}^{a}\right)^{2} \geqslant 0 .
$$

Для наихудшего $\varphi(\cos \varphi=-1)$ неравенство выполняется, если

$$
\left(\mathrm{e}^{a}-1\right)\left(3 \mathrm{e}^{a}+1\right) \geqslant r\left(\mathrm{e}^{a}-1\right)\left[r\left(3+\mathrm{e}^{a}\right)+2\left(\mathrm{e}^{a}-1\right)\right] .
$$

Так как $\mathrm{e}^{a} \geqslant 1$, то правая часть неравенства возрастает с увеличением $r$, а при $r=1$ достигается тождество.

Покажем еще, как надо выбирать постоянную $a$, чтобы модель состояния в экспоненциально-степенном базисе оказалась устойчивой даже для неустойчивой исходной системы.

Из условия

$$
\left(r^{2}-2 r \cos \varphi+1\right) /\left(r^{2}-2 \mathrm{e}^{a} r \cos \varphi+\mathrm{e}^{2 a}\right) \leqslant 1
$$

получим

$$
\mathrm{e}^{a} \geqslant r \cos \varphi \pm(r \cos \varphi-1) \text {. }
$$

Если $\cos \varphi \leqslant 0$, то $\mathrm{e}^{a} \geqslant 1$, а если $\cos \varphi>0$, то $\mathrm{e}^{a} \geqslant 2 r \cos \varphi-1$.

Значит, чтобы получить устойчивую модель состояния в экспоненциально-степенном базисе, надо выбирать постоянную $a$ из условия

$$
a \geqslant \ln \left\{\max \left(2 \operatorname{Re}_{\max }\left[\lambda_{i}(F)\right]-1,1\right)\right\},
$$

где $\operatorname{Re}_{\max }\left(\lambda_{i}(F)\right]$ - максимальная действительная часть среди характеристических чисел матрицы $F(i=1, \ldots, n)$.

Для исследования управляемости и наблюдаемости используем следующий критерий:

пара $(A, B)$, полностью управляема, если только

$$
\operatorname{rank}\left[\lambda_{i}(A) I-A \vdots B\right]=n, \quad i=1, \ldots, n ;
$$

пара $(A, C)$, полностью наблюдаема, если только

$$
\operatorname{rank}\left[\lambda_{i}(A) I-A\right]=n, \quad i=1, \therefore, n
$$

Покажем, что

$$
\operatorname{rank}\left[\lambda_{i}(A) I-A \vdots B\right]=\operatorname{rank}\left[\lambda_{i}(F) I-F \vdots G\right] .
$$

По формулам (19) и (20) с учетом перестановочности матриц $(F-I)$ и $\left(F-\mathrm{e}^{a} I\right)$ получим

$$
\begin{gathered}
\operatorname{rank}\left[\lambda_{i}(A) I-A \vdots B\right]= \\
=\operatorname{rank}\left[\lambda_{i}(A) I-(F-I)\left(F-\mathrm{e}^{a} I\right)^{-1}:\left(1-\mathrm{e}^{a}\right)\left(F-\mathrm{e}^{a} I\right)^{-1} G\right]=
\end{gathered}
$$


$=\operatorname{rank}\left[\frac{\lambda_{i}(A) \mathrm{e}^{a}-1}{\lambda_{i}(A)-1} I-F: \frac{1-\mathrm{e}^{a}}{\lambda_{i}(A)-1} G\right]$

откуда непосредственно следует (23), так как $\left|\lambda_{i}(A)\right|<1$, а по

$$
\lambda_{i}(F)=\left(\mathrm{e}^{a} \lambda_{i}(A)-1\right) /\left(\lambda_{i}(A)-1\right) .
$$

Значит, модель состояния $(A, B, C, D)$ в экспоненциально-степенном базисе будет полностью управляемой, если только исходная система $(F, G, H)$ полностью управляема и $\lambda_{i}(A) \neq 1$.

Аналогично получим

$$
\begin{aligned}
\operatorname{rank}\left[\frac{\lambda_{i}(A) I-A}{C}\right] & =\operatorname{rank}\left[\begin{array}{c}
\frac{\lambda_{i}(A) \mathrm{e}^{a}-1}{\lambda_{i}(A)-1} I-F \\
\frac{1}{\lambda_{i}(A)-1} H
\end{array}\right]= \\
& =\operatorname{rank}\left[\frac{\lambda_{i}(F) I-F}{H}\right] .
\end{aligned}
$$

Значит, модель состояния $(A, B, C, D)$ в экспоненциально-степенном базисе будет полностью наблюдаемой, если только исходная система полностью наблюдаема и $\lambda_{i}(A) \neq 1$.

\section{5. Определение модели состояния в экспоненциально-степенном базисе по импульсной характеристике системы}

Для простоты изложения предположим, что $m=1, x(0)=0$. Тогда импульсная характеристика системы определяется как выходная последовательность $y(t), t=0,1,2, \ldots$, соответствующая входному сигналу

$$
u(t)=\left\{\begin{array}{l}
1, t=0 \\
0, t \neq 0
\end{array}\right.
$$

Найдем соответствующие разложения в экспоненциально-степенном базисе по формуле (11)

$$
\begin{gathered}
y_{k}=\sum_{t=0}^{\infty} \omega(t) \gamma_{k} l_{k}(t) y(t), \\
u_{k}=\omega(0) \gamma_{k} l_{k}(0)=\left(\mathrm{e}^{a}-1\right) / k ! \mathrm{e}^{(k+1) a} .
\end{gathered}
$$

Из предположения $x(0)=0$ и соотношения (23) получим $z_{0}=0$. Теперь мы можем найти последовательность $y_{k}, k=0,1,2, \ldots$, из уравнений состояния (17), (18)

$$
y_{k}=D u_{k}+\sum_{j=0}^{k-1} C A^{k-j-1} B u_{j}
$$

или, учитывая еще (26),

$$
y_{k}=\left(\mathrm{e}^{a}-1\right)\left[\frac{D}{k ! \mathrm{e}^{(k+1) a}}+\sum_{j=0}^{k-1} \frac{1}{j ! \mathrm{e}^{(j+1) a}} C A^{k-j-1} B\right] .
$$

Обозначим

Тогда

$$
h_{k}=\left(\mathrm{e}^{a} y_{k}-y_{k-1}\right) /\left(\mathrm{e}^{a}-1\right) .
$$

$$
h_{0}=D=y_{0} \mathrm{e}^{a} /\left(\mathrm{e}^{a}-1\right),
$$




$$
h_{k}=C A^{k-1} B
$$

т. е. $h_{k}, k=0,1,2, \ldots$, есть ничто нное как марковские параметры модели $(A, B, C, D)$.

Мы можем теперь представить процедуру определения модели состояния в экспоненциально-степенном базисе $(A, B, C, D)$ по импульсной характеристике исходной системы $y(t)$.

1. Находим разложение $y_{k}, k=0,1,2, \ldots$, последовательности $y(t)$, $t=0,1,2, \ldots$, по соотношению $(25)$.

2. Определяем матрицу $D$ по формуле (28).

3. Находим марковские параметры $h_{k}, k=1, \ldots, 2 n-1$, по уравнению (27).

4. Находим методами реализащии модели состояния тройку матрищ $(A, B, C)$ по марковским параметрам $h_{k}, k=1, \ldots, 2 n-1$.

Соотношения (27)-(29), а также представленная процедура остаются в силе и для $m>1$. (21)

Обыкновенную модель состояния можно найти из формул (19)-

$$
\begin{aligned}
& F=(I-A)^{-1}\left(I-\mathrm{e}^{a} A\right), \\
& G=(I-A)^{-1} B, \\
& H=\left(I-\mathrm{e}^{a}\right) C(I-A)^{-1} .
\end{aligned}
$$

\section{6. Заключение}

Модель состояния в экспоненциально-степенном базисе является полноценным представлением дискретной динамической системы. Она имеет те же основные показатели (степень системы, нулевое начальное состояние) и качественные свойства (управляемость, наблюдаемость), что и обыкновенная модель состояния, но при этом характер ее динамических свойств (полюсов системы) можно варьировать в широких пределах выбором постоянной $a$. Так, по желанию можно повысить быстродействие н запас устойчивости модели, а также приобрести устойчивую модель состояния для неустойчивой системы. Последнее обстоятельство может оказаться весьма полезным при синтезе регулятөров и фильтров для неустойчивых объектов.

\section{Л И ТЕ РА Т У РА}

1. Пе ров В. П., Автоматика и телемеханика, № 10, 58-65 (1976).

2. King, R. E., P a ra s kevopoulos, P. N., Int. J. Circuit Theory and Appl., 5, № 1, $81-91$ (1977).

3. Paraskevopoulos, P. N., King, R. E., In: Identification and System Parameter Estimation, Part 2, IV IFAC Symposium, Moscow, 1976, p. 536-543.

4. Kuk k, V., Electron. Lett., 7, № 10, 269-270 (1971).

5. Nurges, U., Ja aksoo, U., In: Control Sciences and Technology for the Progress of Society, VIII IFAC Congress, Kyoto, 1981, 8, p. 56-61.

Ннститут кибернетики

Академии наук Эстонской ССР
Поступила в редакцию 2/II 1982 
DISKREETSE SUSTEEMI OLEKUMUDEL ORTOGONAALSEL BAASIL

On leitud mitmemôõtmelise lineaarse dünaamilise süsteemi uus mudel, nimelt olekuvôrrandid süsteemi sisend- ja väljundsuuruste reaksarenduste vahel V. Perovi $\left.{ }^{[1}\right]$ esitatud diskreetsete ortogonaalșete polünoomide järgi. On saadud lihtsad valemid üleminekuks tavaliselt olekumudelilt uuele mudelile ja vastupidi .ning näidatud, et stabiilse, juhitava ja jälgitava süsteemi olekumudel ortogonaalsete polünoomide baasil on samuti stabiilne, juhitav ja jälgitav. Veelgi enam, sobiva arenduskonstandi valikuga on vōimalik saada stabiilset mudelit ka ebastabiilse süsteemi jaoks.

\section{O. NURGES}

\section{THE STATE EQUATIONS OF DISCRETE TIME SYSTEMS IN AN ORTHOGONAL BASIS}

The problem treated in this paper is the modeling of linear sampled data systems using a new orthogonal system of power polynomials of a discrete-time variable introduced by V. P. Perov $\left[{ }^{1}\right]$

$$
l_{k}(t)=k ! \sum_{j=0}^{h}\left(1-\mathrm{e}^{a}\right)^{j}\left(\begin{array}{l}
k \\
j
\end{array}\right)\left(\begin{array}{l}
t \\
j
\end{array}\right),
$$

where $\left(\begin{array}{l}k \\ j\end{array}\right)$ is the binomial coefficient $a \in[0, \infty)$.

A multivariable dynamical system is considered

$$
\begin{aligned}
x(t+1) & =F x(t)+G u(t), \\
y(t) & =H x(t), \quad t=0,1,2, \ldots,
\end{aligned}
$$

where $x \in R^{n}, u \in R^{m}, y=R^{p}$, and the state equations for the expansion coefficients of input $u(t)$ and output variable $y(t)$ are derived

$$
\begin{aligned}
z_{h+1} & =A z_{h}+B u_{k}, \\
y_{k} & =C z_{k}+D u_{k}, \quad k=0,1,2, \ldots,
\end{aligned}
$$

where $z_{0}=\left(1-\mathrm{e}^{a}\right) x(0)$.

Simple formulas for the transformation of the original state equations to the state equations in the orthogonal basis and vice versa are obtained. Qualitative analysis of the new model revealed that it is stable, controllable and observable if only the original system is stable, controllable and observable. Moreover, if the constant $a$ is chosen according to the inequality

$$
a \geqslant \ln \left\{\max \left(2 \operatorname{Re}_{\max }\left[\lambda_{i}(F)\right]-1,1\right)\right\},
$$

where $\operatorname{Re}_{\max }\left[\lambda_{i}(F)\right]$ is the maximal real part of the eigenvalue $\lambda_{i}, i=1, \ldots, n$, of the matrix $F$, the state equations in the orthogonal basis will be stable even for an unstable original system. At the end of the paper, a partial realization problem is solved to obtain the model in the orthogonal basis from the finite sequence of expansion coefficients. 